\title{
Osteoporosis and Bone Defects in Dentistry: New Drugs and Treatment Options
}

\author{
Mauro Labanca*,1 and Paolo Brunamonti Binello ${ }^{2}$ \\ ${ }^{I}$ Dept. of Dentistry University “Vita Salute” S . Raffaele Hospital, Milan, Italy \\ ${ }^{2}$ Dept. of Specialized Surgery, C. Str. of Dentistry Galliera Hospital, Genoa, Italy
}

\begin{abstract}
First of all we must consider what Osteoporosis is and who is affected by it. We analyze the most common therapies (Biphosfonates, etc.) employed in this field and what their side effects are like ONJ; basically no evidence based of medicine is existing at the moment about ONJ. Patients affected by Osteoporosis and undergoing Biphosfonate therapies that need any oral surgery in general, and implant treatment in particular, could be considered as more risky patients compared with normal patients, but once again not much data is available. We verify if there are some safety precautions to take before treating these patients and if there are some procedures to avoid; considering the important rule that implant treatment should have nowadays for the quality of life of the patients. Finally, we discuss if there are existing or will be present in the future on the market some specific drugs, which would be able to increase the Osteo-integration and bone healing of patients affected by Osteoporosis.
\end{abstract}

Keywords: Osteoporosis, osteonecrosis of the jaw, bone loss, biphosphonates.

\section{INTRODUCTION}

Osteoporosis is a pathology characterized by an alteration of the cellular turn-over level of the bone tissue in which the quota of bone re-absorption overtakes the new bone formation with consequent reduction of bone mass and in absence of alterations of the mineralization in the bones. The main alteration of the cellular turn-over consists in an increase in the osteoclastic activity.

There is evidence that osteoporosis affects the craniofacial and oral structures, although the contribution of osteoporosis in the loss of periodontal attachments, teeth and height of the residual ridge has not been clearly elucidated. It has been shown that systemic osteoporosis affects edentulous mandibles, but not dentate mandibles [1]. Alteration in bone turnover and consequent periodontal problems influence the response to orthodontic forces and this should be taken into consideration when planning orthodontic treatment in osteoporotic patients, especially postmenopausal females or those on chronic medication affecting bone metabolism [2].

The peak of bone mass in both sexes is reached about 35 years of age and lasts a maximal limit for about a decade, during which the turn-over bone mass remains in constant equilibrium: the apposition of newly formed bone, compensates the physiological re-absorption. In the successive stages, we begin to observe and record a para-physiological loss of bone mass, quantifiable in a $0.3-0.5 \%$ per annum. In the female gender, the osteoclastic activity increases inevitably with the reduction of serum concentration of estrogens and with the onset of menopause, certainly however

\footnotetext{
*Address correspondence to this author at the Dept. of Dentistry University "Vita Salute" S . Raffaele Hospital, Milan, Italy; Tel: +3902804246; Fax: +3902864056; E-mail: maurolab@tin.it
}

after 60-years. The peri-menopausal phase of estrogendeficit determines an increase in the seric cytokine (Interleukin-1, Interleukin-6, tumor necrosis factor- $\alpha$, etc.), which predisposes the recruitment, the differentiation, and the activation of the osteoclasts.

We can summarize to the following risk factors the inevitable alterations of the hormonal axis :

- $\quad$ thin constitution and Caucasian race (often associated with phenomena of reduced bone density);

- $\quad$ insufficient dietary supply of Calcium, which leads to a progressive reduction of bone density;

sedentary life, a determining factor in the reduction of bone mass;

tabagism;

alcoholism;

deficiency of Vitamins D2 and D3 (Hypovitaminosis).

The lack of Vitamin D in the population of the world is unanimously recognized by experts, as one of the principal factors of Osteoporosis. It is practically endemic because of the reduction of food income. Needless to say reduced exposure to sunlight is also a crucial factor. The cut-off of Vita$\min \mathrm{D}$ is $30 \mathrm{mg} / \mathrm{ml}$ : inferior values show a lack, which would be opportune to diagnose and treat as a preventative measure. The Vitamin D3 (colecalciferol) derives from the cutaneous synthesis starting with the 7 deidrocholesterol under the action of solar radiations. It may even have an exogenous origin directly from the dietary supply. The Vitamin D2 (ergo-calciferol) comes exclusively from the diet (salmon, sardines, mackerel, tuna, cod liver oil, mushrooms, egg yolks, dairy products, oranges, butter, margarine, cereals, etc.) Since Vitamin D is liposoluble with deposit in the adipose tissue, they undergo two hydrosilations, one in the liver 
in position 25 and another in the kidney in position 1 with formation respectively of $25(\mathrm{OH}) \mathrm{D}$ vitamin (calcifediol) and $1.25(\mathrm{OH}) 2$ Vitamin D (calcitriolol, biologically active). In the D hypovitaminosis, the calcemia is maintained at the expense of a secondary hyper-parathormone.

After the sixth decade of life in both sexes, the osteoblastic activity is reduced in a significant way: the consequent acceleration of the bone re-absorption may produces an increase in the loss of skeletal tissue about ten times more to the physiological annual re-absorption.

The reduction of the bone mass involves the cortical bone as well as the trabecular. From a histological point of view, in fact, a reduction of the thickness of the cortical bone is observed with decrease in the width of the trabecular spongy bone, while the thickness in the lines of osteoid conjunction is preserved. In such a context the quantity of bone mass available to support skeletal structure of the body becomes so fragile that it may reach the level of risk of a spontaneous fracture. Yet, the risk of fracture is directly correlated not so much to the level of osteoporotic re-absorption, but rather, to the density (that is to the quantity of bone for given volume) of the residual bone [3].

Osteoporosis, when not complicated, may remain free of symptoms for years, or even decades; or it may manifest itself with pain of varying intensity in the joints. The advanced forms are often associated with isolated vertebral collapse or fractures. The multiple fractures from vertebral compression, typical of the underlying segment of the eighth thoracic vertebra, causes in the dorsal kyphosis, with time, a cervical lordosis. The consequent functional stress on the dorsal muscles is cause of elevated soreness and persistent inability. Other frequent fractures are tied generally to accidental falls: they are that of the femur, hip and distal radius. Fractures or infractions of other bone segments (costal, etc.), they may even be secondary to occasional trauma of small causes [4].

Osteoporosis can generically be classified as Primary or Secondary types. The following three types of Primary Osteoporosis are:

Juvenile and idiopathic Osteoporosis of the adult (rare and prevailing in pediatric age);

Type I Osteoporosis, or Menopausal (can be diagnosed in both sexes among the 51 and the 75 years of age following precocious menopause or castration);

Type II Osteoporosis, or Regressive, or Senile (can be diagnosed in both sexes after the sixth decade of life).

\section{PRIMARY OSTEOPOROSIS}

Primary type Osteoporosis occurs in a relatively small subset of postmenopausal women who are 51 to 65 years of age. Less frequently, a similar syndrome occurs in men of comparable age.

This type of osteoporosis is associated with postmenopausal gonadal deficiency and/or is related to a senile decrement of adrenal androgen production [1,5].

\section{SECONDARY OSTEOPOROSIS}

Secondary types of Osteoporosis have a statistical incidence of less than $5 \%$ of all the osteoporosis and can be recognized from the principal following varying symptoms:
"Iatrogenic (corticosteroids, ethanol, tobacco, barbiturates, heparin, etc.)

"Consequent endocrine gland disorders (hyperadrenalism, Hyperparathyroidism, Hypogonadism, diabetes, etc.).

"Consequent on systemic different pathologies (chronic obstructive renal insufficiency, hepatopathies, broncho-pneumopathy, various rheumatoid arthritis, sarcoidosis, neoplasias, etc).

\section{LABORATORY ANALYSES}

The instrumental diagnosis of osteoporosis requires radiological investigations and measurements of bone mineral content.

The laboratory analyses contemplate:

Calcium and Phosphorus quantification in serum;

electrophoresis of serum proteins;

speed of erythrosedimentation;

alkaline serum phosphatase (increase in presence of fractures);

dosing of the serum parathormon (PTH);

Osteocalcine serum;

Calciuria;

Urinary excretion of peptides containing Hidrossyproline and of the Pyridin peptide.

\section{RADIOLOGICAL INVESTIGATIONS}

Quantitative computerized axial tomography (QCT)

Computerized bone mineralometry; called even Densitommetry (or Assorbimmetry): to perform a double radiation (DPA); to single radiation (SPA); digital XRays (DEXA)

The objectives of the treatment of the Osteoporosis are to prevent fractures and to contain the algical symptoms with respect to their function.

Prevention and therapy of minor case histories are causal and pharmacologic.

The principal pharmacological drugs for the therapy of Osteoporosis are listed below (also in order by aggressiveness):

Calcium (1,500 mg per day)

Vitamin D2 and Vitamin D3 (altogether 400 international units per day)

Sodium Fluoride (50 mg per day)

Parathormone (to daily dosing, low and intermittent)

Salmon calcitonin (200 international units per day for inhalation; or 100 international units per day intravenous (i.v.)

Strontium ranelate

Bisphosphonates (with different types of dosage according to the molecule employed). 


\section{BISPHOSPHONATES}

The Bisphosphonate (BP-Bisphosphonate acid) includes a vast group of molecules structurally analogous to the inorganic pyrophosphate, physiologically present in serum and urine. The only structural difference consists in the substitution of the atom of oxygen in central position with one of Carbon.

Therefore, because of the presence of this atom of carbon, that increases significantly the structural stability, these molecules have the advantage, in comparison with the pyrophosphate, to withstand the enzymatic hydrolysis of the organic Pyrophosphates. As well, the BPs introduce elevated heat resistance to the chemical reagents and are able to form a molecular complex non-soluble mixing themselves to metal ions.

Fundamentally three generations of BPs exist. Ca-O6-P$\mathrm{OH}$ is the precursor. Such classification of generational type, based on variations of the chemical structure, came from the demand to study the evidence of its power and to underline from it the different mechanisms of molecular action.

1. Clodronate belongs to the first generation (2CL/CH4O6-P2) as well as Tiludronate (CL/CH4-O6-P2-S-R), they result already be ten times more powerful than Etidronate.

2. Pamidronate (3-aminohidroxypropylidenebis - disodium salt 9CL/C3-H11-N-O7-P2.2N) represents the basic molecule of the second generation and the presence of an amidogen makes it ten times more powerful than Clodronate and Tiludronate [6].

3. The third generation of Biphophonates is, finally, characterized by the addition of a methylic group (besides minor variables) to the $\mathrm{N}$ alkylic chain.

The following belongs to this generation:

Alendronate (1-hidroxy-4-aminobutilene-1, 1-biphosphonic acid /C4-H12-N-O7-P2/C, H, N, P ), that is 100 to 1,000 times more powerful than its precursors;

Ibandronate (1-hidroxy-3-methylpentylaminopropilene-1, 1-second-bisphosphonic acid (C7-H19-N-O7$\mathrm{P} 2$ ), that is after 1.000 to 10.000 times more powerful than its precursors;

Zolendronate (1-hidroxy-2-1H imidazol-1-yl-second ethilidene-9CL/C6-H10-N2-O7-P2), this one is over 10,000 times more powerful than any of its precursors [6].

From the analysis of aforesaid classification the differences of molecular potentiality appear evident. They are linked also to the different mechanism of action among BPs of the first generation and Amino-BPs of the second and third generation. In fact, also if the complete mechanism of action of the BPs is not still entirely clear, we have observed that, in vitro, they bind themselves firmly to the solid phase of the Idroxyapatite (Ca10-PO4-6OH2), preventing its dissolution. Such action comes true in vivo due to the bond between the pharmacological molecules and the de-mineralized component of the bone tissue.

BPs of the first generation become metabolized at a cellular level in a mixture capable of competing with the
Adenosintriphosphate (ATP) - responsible for the energetic metabolism of the cell-introducing as analogous not hydrolizable: it follows that the osteoclast ends in an energetic exhaustion and apoptosis. The Amino-BPs of the second and third generation, are able to directly stop the cellular enzyme farnesyl diphosphate-syntetase, since they are part of the metabolic pathway of Mevalonates: this blockage determines the failed synthesis of some essential metabolites to allow the prenilation of the proteins, which are in the external cell membrane of the osteoclasts. Such action interferes in the proliferation and transmission of the signals in the cell and in a morphological alterations; this causing its death (apoptosis). The interaction among BPs and bone inorganic component, doesn't influence either the osteogenesis, or the mechanical ownership of the same bone; but inhibits the precursors of the osteoclasts, halting the mechanisms of reabsorption.

Thanks to the qualities of these BPs, experts have found for several years now, these drugs the effectiveness treatment for the care of all the primitive and secondary pathologies that involves alterations of bone metabolism (osteoporosis, Paget's disease, primitive and secondary neoplasias, etc).

The pharmacokinetics is conditioned by the fact that, dealing with insoluble molecules, they diffuse with difficulty across the biological barriers: if administered for enteric way the absorption is limited to the small intestine with assimilation around $5-10 \%$ of the general dose. The serum emivita is reduced, since the molecule is rapidly excreted through the renal system; however, it remains in the mineralized component of the bone tissue even for many years after it is taken. They are also constantly released in the haematic stream and over 54\% of the circulating molecules bind to the plasmatic proteins. Such percentage value increases when the concentrations of Calcium becomes particularly high. The calcium amounts of serum, in the presence of bone pathological reabsorbtion, is recalled by the mineral bone exposed in the areas of lysis, that become inhibited.

Clinical experimental studies conducted on oncological patient with skeletal metastasis of lytic type has confirmed beneficial advantages when BPs are administered [7]. There has been a considerable reduction of the lytic phenomena and an attenuation of the algical symptoms correlated to them. The toxicity of the Bisphophonates seems to have a direct effect on the parenchyma regions mostly vascularized, like skeletal muscle, cardio-circulatory apparatus and renal system. In patients suffering from serious renal insufficiency (clearance of the creatinine $<30 \mathrm{ml} /$ minute) the plasmatic concentration of BPs may become three times superior to that of patients with renal physiological functionality. In the last years, then, cases have been reported in medical literature of Osteonecrosis of the maxillary bone correlated to the treatment of BPs [8].

\section{OSTEONECROSIS OF THE JAW (ONJ) CORRE- LATED TO THE TREATMENT WITH BISPHOS- PHONATES}

Statistically, conflicting data still exists about the incidence of this complication, correlated to the treatment with BPs, that is at the present time considered a rare and late event [9]. The values of tests and incidence reported in literature vary from $0.8 \%$ to $12 \%$ : such variability depends on the 
different methods used by the authors about the collection and the classification of the data, but even from the nonhomogeneous quality of the samples taken. Such lack of homogeneity stems from the objective impossibility to establish analogous clinical requisites in order to form a basis for the standardization of treatment.

The etiopathogenesis or beginning of the ONJ is of avascular and osteomyelitic type [10-12]. Be it in vivo or in vitro, antineo - angiogenetic action of the BP is demonstrated, even if the molecular mechanism that induces the lesion has yet to be identified. Mashiba and collaborators maintain that such molecules cause damage to the micro-vascular system due to the accumulation of damaged osteoclasts in the little capillaries [13]. Kapitola and collaborators, in a study conducted on animals, formulated the hypothesis that such molecules reduce the hematic flow to the medullary bone, interacting with the hormones and growth factors [14].

The majority of the lesions is found at the level of the maxillary (and in particular to the lower jaw). The majority of the authors concur for the following reasons:

- $\quad$ the jaw is subject to an elevated cellular turn- over;

- $\quad$ the masticatory function induces micro-traumas (micro-fractures) constantly;

the presence of a bacterial flora in the oral cavity helps the onset of complications of septic type.

There are also predisposing factors to consider:

Poor oral hygiene,

Excessive Smoking and Alcoholism;

Decubitus from defective or incongruous prosthetics;

Foci in the periodontal pockets;

Damage due to cavities, granulomas or apical lesions;

Systemic persistent affections (mellitus diabetes, Immuno-depression, etc);

Oral invasive surgery (dental extractions, etc.) above all if not supported by antibiotic prophylaxis.

There are different criteria and classifications of ONJ [15]. Because of the clinical experience of the authors this classification which they have set up has assisted them in obtaining positive and effective results in the diagnostic profile and descriptive areas. They are able to prognosticate, quite accurately because their findings are based exclusively on the location and extension of the lesion in object [16]:

I STAGE: ONJ with extension < $1 \mathrm{~cm}$; (Fig. 1).

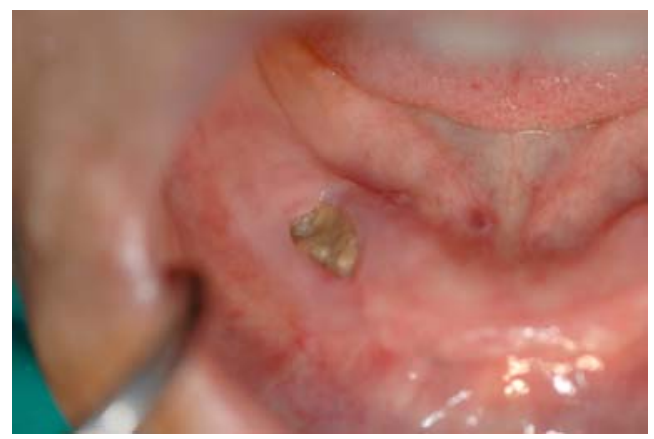

Fig. (1). ONJ with extension < 1 CM ( STAGE 1).
I STAGE A - involvement of important anatomical structures (like nerves or vessels) is absent;

I STAGE B - adjacent structures are involved;

II STAGE : ONJ with extension =1-4 cm; (Fig. 2).

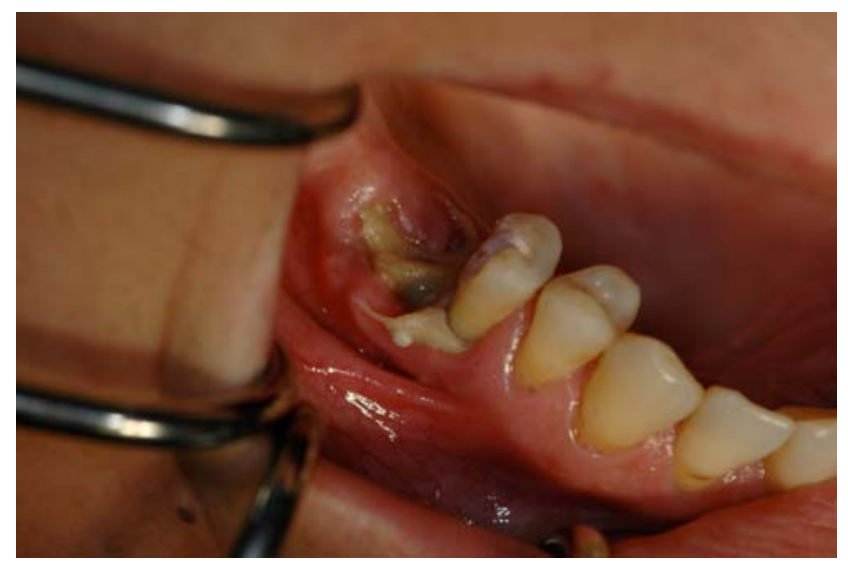

Fig. (2). ONJ with extension =1-4 CM (STAGE 2).

II STAGE A - absence of involvement of adjacent important anatomical structure;

II STAGE B - adjacent anatomical structures are involved;

III STAGE: ONJ with extension > $5 \mathrm{~cm}$; (Fig. 3).

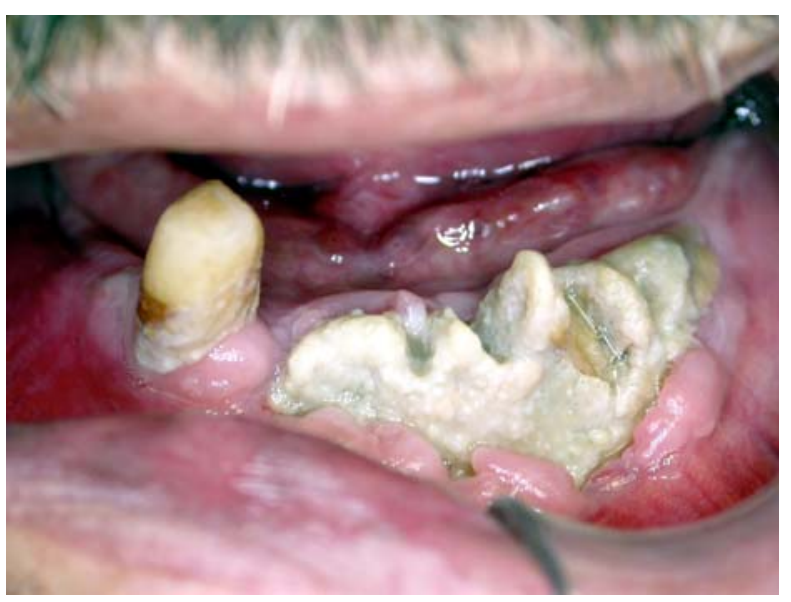

Fig. (3). ONJ with extension > 5 CM (STAGE 3).

IV STAGE: ONJ with the involvement in toto of only one of the maxillas; (Fig. 4).

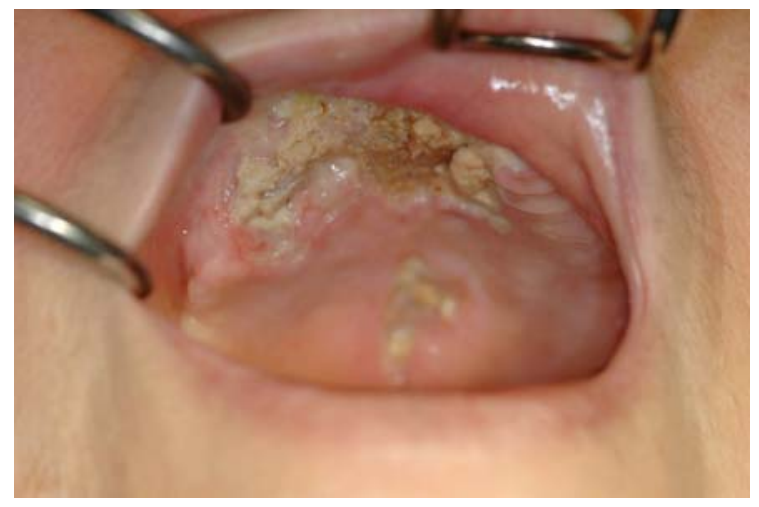

Fig. (4). ONJ with involvment in toto only of one of the Jaw (STAGE 4). 
V STAGE: ONJ with involvement in toto of both the jaws.

The diagnosis of ONJ is substantially clinical and corresponds to the presence of bone persistent exposure for more than 8 weeks in a patient submitted to treatment with BPs and with a negative case history for radiotherapy of the maxillaries. From a radiographic point of view, the clinical situation is similar to that of bacterial osteomyelitis or of Osteoradionecrosis. The same can be said for the histologi$\mathrm{cal} / \mathrm{bacteriological}$ point of view, when bone necrosis is found with bacterial colonization [16,17].

There is a state of the art agreement among the Authors about the usefulness of preventing ONJ from BPs by eliminating the pre-disposing co-factors and submitting all those candidates for BPs to a meticulous Odontosmatologic examination (in order to avoid a pre-existing dental infection). There still does not exist today a procedural set of rules to treat such complications, which can adversely affect the quality of life. Unlike the traditional ONJ, those ones that are linked to treatment with BPs manifest a strong tendency not to react to the traditional treatments (targeted antibiotic therapies), or to worsen, resulting in negative result if treated with a radical surgical approach [17].

The scientific community concurs, if it is considered essential, and if the systemic conditions of the patient allow it, to perform a less invasive surgical approach. Thus, the introduction of the concept of "Soft Oral Surgery", which foresees the realization of osteotomies done by piezo-surgery and limited to the bony necrotic segments vulnerable into the oral cavity. Procedures exist, but are still in an experimental phase-that consider the pre-treatment and post-surgical use of cycles of Ozone therapy, Oxygen therapy and Regenerative therapy.

\section{ODONTOSTOMATOLOGIC APPROACH TO THE PATIENT IN TREATMENT WITH BIPHOSPHO- NATES FOR OSTEOPOROSIS}

The greatest part of the maxillary ONJ correlated to treatment with BP mentioned in literature concerns cases of patients with secondary oncological afflictions affecting the bones and undergoing therapy with molecules of second and third generation of elevated dosage and administered in a parenteral manner. This compels the majority of the authors to consider such events as exceptional complications in non oncological patients, treated with the recommended dosage for the prevention and care of the Osteoporosis. Even in aforesaid clinical situation, considerable importance is given to the systemic conditions of the patient in observation.

With the intent to create uniformity in the Odontostomatological protocols for the management of the patient in treatment with BP, the Italian Group for the Biphosphonate Study (G.I.B.I.S.) created and elaborated in 2007 a classfication of groups of patients with the relative indications to the treatment:

\section{Group A}

Patients that have been treated with Bisphophonate (orally somministration (o.s.) or i.v.) for a period not longer than 3 months. After a congruous period of about 4-6 weeks from the date of the last intake, such patients are able to undergo any dental treatment (urgent or routine) inclusive of implantology under suitable antibiotic dosage;

\section{Group B}

Patients undergoing treatment with BPs (per os or i.v.) over than 3 months (even for several years) who have never presented symptoms of ONJ. In this case there is a tendency to limit oral surgery (including the normal extractions) to those absolutely necessary and after a waiting period of at least 3-6 months has elapsed from the last dosage and with suitable antibiotic treatment. This waiting period doesn't extinguish at all danger of an onset of ONJ, but reduces the risk.

\section{Group C}

Patients in treatment with biphophonates (or those who have previously been under treatment), that are already affected with ONJ. In this case it is advisable to maintain a conservative attitude as much as possible, avoiding or reducing the need for surgery to include only essential, minimal invasive procedures.

According to the above-mentioned classification the surgical procedure and the prosthetic-implant treatments do not present any contra-indications in patients belonging to GROUP A. It is also clear that this approach is negative in every way to those patients classified as GROUP C, therefore inadvisable to consider. The debate is still open regarding the possibility of treatment in GROUP B patients. A majority of authors thinks that in order to pursue an Evidence Based of Medicine - the creation of a random survey with post operative follow up not less than five years would be a positive step. The personal opinion of the authors is, considering the heterogeneous typology of the patients, not to ignore in any way the systemic conditions and the clinical history of the individual case.

\section{CONCLUSIONS}

The data reported in literature in reference to the clinical evolution of Osteonecrosis correlated to the treatment with BPs are still partials and controversial today. Initially it was concluded that the possibilities of recovery were extremely few and there was no known, specific, suitable treatment to restore the lesions in question with restitutio ad integrum. The authors are aware of the actual demand to distinguish among two different concepts of recovery, not antithetical (against each other) but different. The first is represented by a histological recovery, complete and absolute by definition, that in this case can only be followed up but not actively pursued. The second is constituted by a concept of clinical recovery, which can be verified with routine radiodiagnostics and objective local examinations.

Currently there are therapeutic protocols, though still in experimental stage; they produce such results, which serve as a stimulus for us and others to continue the research. They open new hopes above all in cases of patients who are Osteoporotic. We have observed resolutions often with only a conservative medical therapy, or with mini-invasive surgery.

In great expectations, then, of a more profound knowledge about the evolution of this iatrogenic complication, it would be opportune to:

Stimulate collaboration among multidisciplinary teams ready to synergize their individual clinical experiences and to encourage dialogue and collabora- 
tion between the different specialist fields of Medicine (Oncology, Physiatrics, Endocrinology, Geriatrics, Internal Medicine, Orthopedics, Urology, Odontostomatology, Maxillofacial Surgery, etc.).

- Preventive dental consultations for all the patients who are candidates for the treatment with BPs and the removal of any possible infections in the oral cavity.

- $\quad$ Better evaluate the clinical and medical histories (past as well as present) during the consultation to the dental specialist.

\section{ABBREVIATIONS}

$$
\begin{aligned}
& \text { BP }=\text { Bisphosphonates } \\
& \text { ONJ }=\text { Osteonecrosis of the jaw } \\
& \text { BRON }=\text { Bisphosphonates Related Osteonecrosis } \\
& \text { GIBIS }=\text { Italian group for the Biphosphonates study } \\
& \text { i.v. } \quad=\text { Intravenous } \\
& \text { o.s. } \quad=\text { Oral somministration }
\end{aligned}
$$

\section{REFERENCES}

[1] Erdoğan, O.; Shafer, D.M.; Taxel, P.; Freilich, M.A. A review of the association between osteoporosis and alveolar ridge augmentation. Oral Surg. Oral Med. Oral Pathol. Oral Radiol. Endod., 2007, 104, 738.e1-13.

[2] Sidiropoulou-Chatzigiannis, S.; Kourtidou, M.; Tsalikis, L. The effect of osteoporosis on periodontal status, alveolar bone and orthodontic tooth movement. A literature review. J. Int. Acad. Periodontol., 2007, 9, 77-84.

[3] Di Monaco, M.; Di Monaco, R.; Mautino, F.; Cavanna, A. Femur bone mineral density,age and fracture type in 300 hip-fractured women. Aging Clin. Exp. Res., 2002, 14, 47-51.

[4] Di Monaco, M.; Vallero, F.; Di Monaco, R.; Mautino, F.; Cavanna A. Functional recovery and length of stay after hip fracture in patients with neurologic impairment. Am. J. Phys. Med. Rehabil., 2003, 82, 143-8;

[5] Riggs, BL.; Melton, L.J. 3rd. Evidence for two distinct syndromes of involutional osteoporosis. Am. J. Med., 1983, 75, 899-901.
[6] Marx, RE.; Pamidronate (Aredia) and zoledronate (Zometa) induced avascular necrosis of the jaws: a growing epidemic. J. Oral Maxillofac. Surg., 2003, 61, 1115-7.

[7] Berenson, J.R.; Rosen, L.S.; Howell, A.; Porter, L.; Coleman, R.E.; Morley, W.; Dreicer, R.; Kuross, S.A.; Lipton, A.; Seaman, J.J. Zoledronic acid reduces skeletal-related events in patients with osteolytic metastases. Cancer, 2001, 91, 1191-200. Erratum in: Cancer., 2001, 91, 1956.

[8] Ripamonti, C.I.; Maniezzo, M.; Campa, T,; Fagnoni, E.; Brunelli, C.; Saibene, G.; Bareggi, C.; Ascani, L.; Cislaghi, E;. Decreased occurrence of osteonecrosis of the jaw after implementation of dental preventive measures in solid tumour patients with bone metastases treated with bisphosphonates. The experience of the National Cancer Institute of Milan. Ann. Oncol., 2009, 20, 137-45.

[9] Marx, RE.; Sawatari, Y.; Fortin, M.; Broumand, V. Bisphosphonate-induced exposed bone (osteonecrosis/osteopetrosis) of the jaws: risk factors, recognition, prevention, and treatment. J. Oral Maxillofac. Surg., 2005, 63, 1567-75.

[10] Brunamonti, B. P.; Decensi, A.; Caroti, C.; Merlini, A.; Del Buono, V.; De Angelis, D.; Balbi, Q. Osteonecrosi avascolare dei mascellari con osteomielite secondaria in pazienti sottoposti a trattamento con bifosfonati. Quintessenza Internazionale, 2007, 1, 49-58.

[11] Brunamonti, B. P.; Giannattasio, A.; Garibaldi, J.; Balbi, P. Osteonecrosi avascolare dei mascellari correlata ai bifosfonati (BRON-ONJ). Criterio di classificazione originale e prospettive terapeutiche. Quintessenza Internazionale, 2008, 3, 33-45.

[12] Migliorati, C.A. Bisphosphanates and oral cavity avascular bone necrosis. J. Clin. Oncol., 2003, 21, 4253-4.

[13] Mashiba, T.; Hirano, T.; Turner, C.H.; Forwood, M.R.; Johnston, C.C.; Burr, D.B.; Suppressed bone turnover by bisphosphonates increases microdamage accumulation and reduces some biomechanical properties in dog rib. J. Bone Miner. Res., 2000, 15, 613-20.

[14] Kapitola, J.; Zák, J.; Lacinová, Z.; Justová V. Effect of growth hormone and pamidronate on bone blood flow, bone mineral and IGF-I levels in the rat. Physiol. Res., 2000, 49(Suppl), S101-6.

[15] Ficarra, G.; Bennati, F.; Rubino, I.; Vannucchi, A.; Longo, G.; Tonelli, P.; Pini Prato, G. Osteonecrosis of the jaws in periodontal patients with a history of bisphosphonates treatment. J. Clin. Periodontol., 2005, 32, 1123-8

[16] Ruggiero, S.L,; Mehrotra, B., Rosenberg, T.J.; Engroff, S.L. Osteonecrosis of the jaws associated with the use of bisphosphonates: a review of 63 cases. J. Oral Maxillofac. Surg., 2004, 62, 527-34.

[17] Ruggiero, S.L.; Fantasia, J.; Carlson, E. Bisphosphonate-related osteonecrosis of the jaw: background and guidelines for diagnosis, staging and management. Oral Surg. Oral Med. Oral Pathol. Oral Radiol. Endod., 2006, 102, 433-41.

(C) Labanca and Brunamonti Binello; Licensee Bentham Open .

This is an open access article licensed under the terms of the Creative Commons Attribution Non-Commercial License (http://creativecommons.org/licenses/by-nc/3.0/) which permits unrestricted, non-commercial use, distribution and reproduction in any medium, provided the work is properly cited. 